\title{
Banco de dados da comunidade de mamíferos de médio e grande porte da Estação Ecológica do Taim, Rio Grande do Sul, Brasil.
}

Medium and large mammal community dataset of the Taim Ecological Station, Rio Grande do Sul, Brazil.

\author{
Alex Bager ${ }^{{ }^{*}}$; Érika P. Castro ${ }^{2}$
}

\section{RESUMO}

O banco de dados inclui dados de levantamentos de armadilhas fotográficas de mamíferos de médio e grande porte, realizados na Estação Ecológica de Taim, uma unidade de conservação federal no sul do Brasil. Todas as informações foram registradas entre março de 2002 e maio de 2003 como parte do projeto Estrada Viva, que avaliou os efeitos da estrada sobre a biodiversidade nesta área protegida. Foram instaladas armadilhas fotográficas em quatro áreas com diferentes graus de impactos antrópicos (próximo e distante da rodovia) e tipo de vegetação (campo e floresta). Foram identificadas 13 espécies de mamíferos de médio e grande porte; 11 nativas, uma exótica e outra doméstica. As espécies foram: Capivara (Hydrochoerus hydrochaeris; $n=481$ ), Cachorro-do-campo (Lycalopex gymnocercus; $n=68$ ), Cachorro-do-mato (Cerdocyon thous; $n=51$ ), Ratão-do-banhado (Myocastor coypus; $n=29$ ), Tatu-galinha (Dasypus novemcinctus; $n=24$ ), Mão-pelada (Procyon crancrivorus; $n=7$ ), Zorrilho (Conepatus chinga; $\mathrm{n}=6$ ), Lebre (Lepus capensis; $\mathrm{n}=5$ ); Lontra (Lontra longicaudis; $\mathrm{n}=4$ ); Gato-do-mato-grande (Leopardus geoffroyi; $\mathrm{n}=4$ ); Gambá-de-orelhas-brancas (Didelphis albiventris; $\mathrm{n}=3$ ); Gato doméstico (Felis catus; $\mathrm{n}=2$ ); Tatu-peludo (Euphractus sexcinctus; $\mathrm{n}=2$ ). Os dados permitem avaliar os efeitos da paisagem e da presença de estradas na composição da comunidade faunística da região.

Palavras-chave: Área Protegida; Mamíferos; Armadilha fotográfica; Zona de Efeito; Ecologia de Estradas.

\footnotetext{
${ }^{1}$ Universidade Federal de Lavras, Centro Brasileiro de Ecologia de Estradas, Lavras, Brasil. abager@ecoestradas.org

2 Universidade Federal de Lavras, Programa de Pós-graduação em Tecnologias e Inovações Ambientais, Lavras, Brasil.
} 


\section{ABSTRACT}

This dataset includes data from camera trap surveys of mammals in Taim Ecological Station, a federally protected area in southern Brazil. All information included in the dataset has been recorded between March 2002 and May 2003 as part of Estrada Viva project, which assessed the effects of the road on biodiversity in this protected area. Camera traps were installed in four areas with different degrees of anthropic impacts (near and far from the highway) and vegetation type (grassland and forest). Thirteen medium and large mammal species were identified, 11 native, one alien, and other domestic. The species were: Capybara (Hydrochoerus hydrochaeris; $\mathrm{n}=481$ ), Pampa fox (Lycalopex gymnocercus; $n=68)$, Crab-eating fox (Cerdocyon thous; $n=51$ ), Nutria (Myocastor coypus; $n=29$ ), Nine-banded armadillo (Dasypus novemcinctus; $n=24$ ), Crab-eating raccoon (Procyon crancrivorus; $n=7$ ), Molina's hog-nosed skunk (Conepatus chinga; $\mathrm{n}=6$ ), Cape hare (Lepus capensis; $\mathrm{n}=5$ ); Neotropical otter (Lontra longicaudis; $n=4$ ); Geoffroy's cat (Leopardus geoffroyi; $n=4$ ); White-eared opossum (Didelphis albiventris; $n=3$ ); Domestic cat (Felis catus; $n=2$ ); Six-banded armadillo (Euphractus sexcinctus; $n=2$ ). The data allow us to assess the effects of the landscape and the presence of roads on the composition of the faunal community in the region.

Keywords: Protected Area; Mammals; Camera trap; Effect Zone; Road Ecology.

\section{IMPORTÂNCIA DOS DADOS}

- Avalia o impacto potencial das estradas sobre comunidades de mamíferos de médio e grande porte;

- Fornece dados da abundância e riqueza de mamíferos de médio e grande porte da Estação Ecológica Taim;

- Auxilia no planejamento e no manejo de áreas protegidas no bioma Pampa, no Brasil;

- Os dados podem ser úteis para estudar a relação entre a mortalidade de animais silvestres nas estradas e a abundância de diferentes espécies.

\section{MATERIAL E MÉTODOS}

Este artigo apresenta registros de mamíferos de médio e grande porte, coletados na Estação Ecológica de Taim, localizada na cidade do Rio Grande, Rio Grande do Sul, Brasil. Os levantamentos fizeram parte de um projeto maior (Projeto Estrada Viva), que avaliou a mortalidade de animais silvestres na rodovia BR 471, que cruza a unidade de conservação.

\section{Área de estudo}

A Estação Ecológica de Taim (ESEC Taim) (32。32' 14.44"S; 52。32' 18.67"W) (Fig. 1) é uma unidade de conservação federal, localizada na planície costeira do estado do Rio Grande do Sul, Brasil. A ESEC Taim apresenta uma área de 33.818 ha, com o relevo baixo e plano, e as áreas úmidas são predominantes, embora campos abertos e dunas estejam presentes. O clima é ameno, com um inverno frio e chuvoso e um verão quente e seco. A precipitação média anual é de $1100 \mathrm{~mm}$, e a temperatura média é de $18{ }^{\circ} \mathrm{C}$ (NIMER, 1989).

A ESEC Taim é afetada pela BR 471, uma estrada federal pavimentada, com 2 pistas e um volume médio de tráfego diário de 247 veículos e uma velocidade média de $80 \mathrm{~km} / \mathrm{h}$ (BAGER, 2006). A rodovia afeta diretamente a 
unidade em pouco mais de 15 quilômetros, tangenciando e cortando sua poligonal em diferentes trechos. Desde 1998 a ESEC Taim possui um conjunto de medidas de mitigação, constituído por passagens de fauna, cercas, redutores de velocidade e mata-burros. Bager e Fontoura (2013) avaliaram a eficiência destas medidas na redução dos atropelamentos e demonstraram um sucesso parcial para algumas das espécies mais afetadas.

Figura 1. Mapa da Estação Ecológica do Taim e dos diferentes pontos de amostragem na Unidade e/ou seu entorno direto. F - floresta; C - campo; P - perto; L - longe.

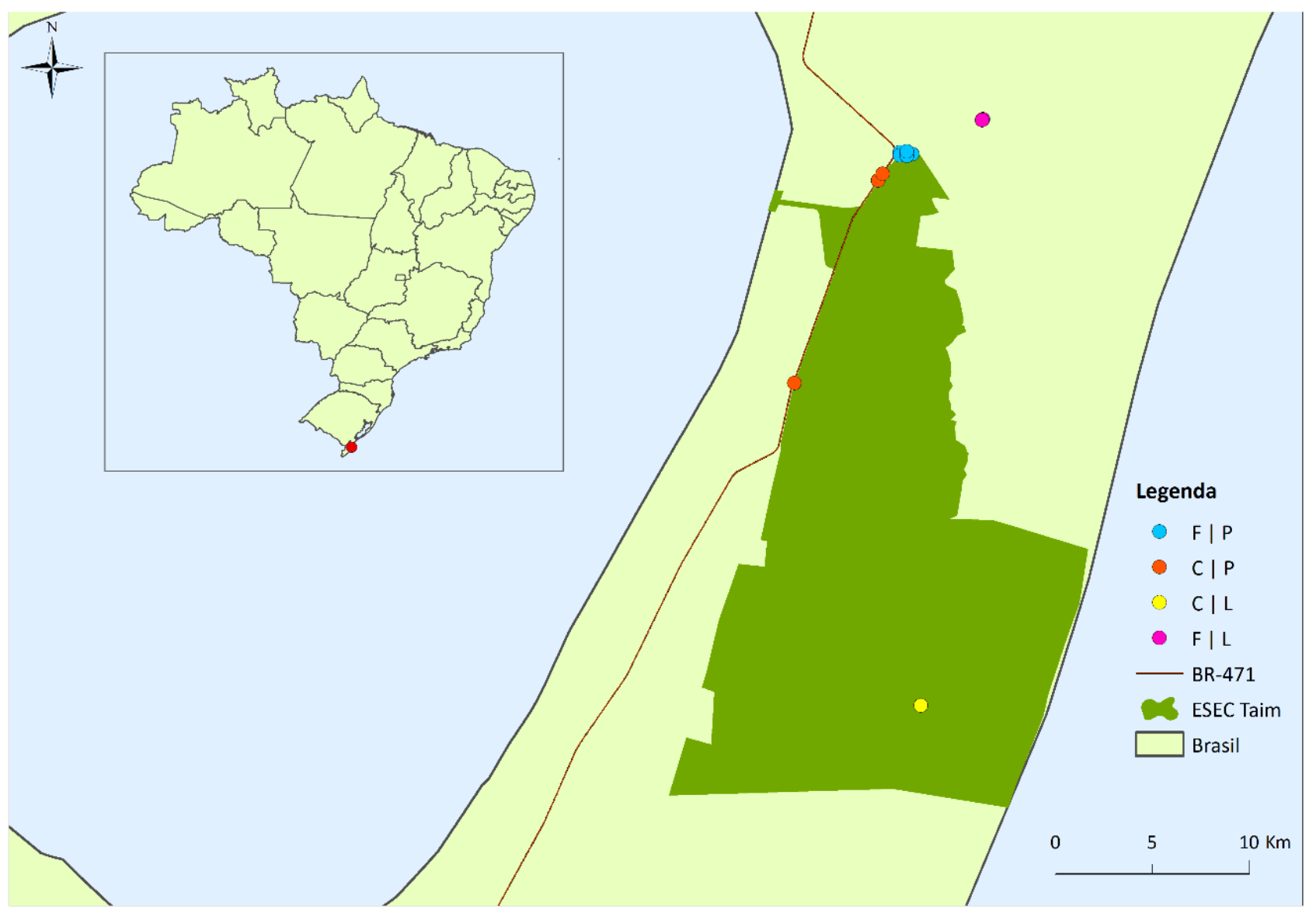

\section{Coleta de dados}

As coletas foram realizadas entre março de 2002 e maio de 2003 utilizando armadilhas fotográficas da marca Tigrinus, totalizando 4.440 horas de esforço amostral. Foram selecionadas 11 áreas de amostragem considerando duas variáveis: (1) tipo de cobertura vegetal (campo e floresta) e (2) distância da estrada (perto e longe). Áreas definidas como longe estavam a, no mínimo, 1 quilômetro da rodovia. Cinco armadilhas fotográficas monitoraram cada local durante diferentes períodos. A Tabela 1 apresenta os dados do levantamento e seus respectivos esforços de amostragem. 
Tabela 1. Dados das áreas de amostragem de mamíferos de médio e grande porte em diferentes locais da Estação Ecológica de Taim. Sessão: a sequência de amostras; Instalação: data de instalação das armadilhas das câmeras; Remoção: data da remoção: Centroide (Lat, Lon): o ponto central de distribuição das câmeras durante a Sessão; Área: F | L (Floresta e Longe), F | P (Floresta e Perto), C | L (Campo e Longe), C | P (Campo e Perto); Esforço: esforço de amostragem (horas) em uma Sessão; Esforço total: Esforço total de amostragem (horas) para um conjunto de Sessões em uma mesma classe de amostragem

\begin{tabular}{cccccccc}
\hline Sessão & Instalação & Retirada & Centroide (Lat) & Centroide (Lon) & Área & Esforço & Esforço Total \\
\hline 5 & $28 / 09 / 2002$ & $30 / 09 / 2002$ & $-32,521339$ & $-52,496899$ & $\mathrm{~F} \mid \mathrm{L}$ & 240 & 720 \\
8 & $15 / 12 / 2002$ & $19 / 12 / 2002$ & $-32,52172$ & $-52,497427$ & $\mathrm{~F} \mid \mathrm{L}$ & 480 & \\
4 & $31 / 08 / 2002$ & $02 / 09 / 2002$ & $-32,537519$ & $-52,52978$ & $\mathrm{~F} \mid \mathrm{P}$ & 240 \\
6 & $04 / 11 / 2002$ & $6 / 11 / 2002$ & $-32,536612$ & $-52,535582$ & $\mathrm{~F} \mid \mathrm{P}$ & 240 \\
7 & $12 / 12 / 2002$ & $14 / 12 / 2002$ & $-32,537964$ & $-52,535582$ & $\mathrm{~F} \mid \mathrm{P}$ & 240 \\
10 & $22 / 03 / 2003$ & $23 / 03 / 2003$ & $-32,538314$ & $-52,532314$ & $\mathrm{~F} \mid \mathrm{P}$ & 120 \\
11 & $03 / 05 / 2003$ & $16 / 05 / 2003$ & $-32,536128$ & $-52,532314$ & $\mathrm{~F} \mid \mathrm{P}$ & 1560 \\
1 & $16 / 03 / 2002$ & $17 / 3 / 2002$ & $-32,549684$ & $-52,5456$ & $\mathrm{C} \mid \mathrm{P}$ & 120 \\
2 & $06 / 04 / 2002$ & $8 / 4 / 2002$ & $-32,546571$ & $-52,543502$ & $\mathrm{C} \mid \mathrm{P}$ & 240 \\
3 & $04 / 05 / 2002$ & $7 / 5 / 2002$ & $-32,64386$ & $-52,584519$ & $\mathrm{C} \mid \mathrm{P}$ & 360 \\
9 & $15 / 02 / 2003$ & $20 / 2 / 2003$ & $-32,793741$ & $-52,525825$ & $\mathrm{C} \mid \mathrm{L}$ & 600 \\
\hline
\end{tabular}

\section{DESCRIÇÃO DE DADOS}

Foram identificadas 13 espécies e 681 registros nas amostragens, sendo 11 nativas silvestres, uma exótica e uma doméstica (Tabela 2). A espécie mais abundante foi a capivara (Hydrochoerus hydrochaeris), seguida pelas duas espécies de canídeos que habitam a região, Lycalopex gymnocercus e Cerdocyon thous, respectivamente com 68 e 51 registros fotográficos. As áreas de mata tiveram uma maior riqueza do que as áreas de campo, já as áreas diretamente associadas à rodovia tiveram uma maior abundância do que às distantes, sobretudo devido ao elevado número de capivaras nestas áreas.

\section{Banco de Dados}

O banco de dados (Dataset Bager \& Castro) é um arquivo Excel, constituído por uma única aba (Dados Brutos) com 14 colunas, sendo elas:

- Período: caracteriza-se por um período de amostragem, o qual reúne um conjunto de armadilhas fotográficas instaladas em uma área geográfica com uma determinada característica de paisagem e proximidade com a rodovia.

- Instalação: data de instalação de uma determinada armadilha fotográfica.

- Retirada: data de retirada de uma determinada armadilha fotográfica.

- Máquina: código dado a uma armadilha fotográfica específica e que permaneceu do início ao fim das coletas de dados.

- Filme: as armadilhas fotográficas não eram digitais e utilizavam filmes. Conforme o 
número de registros, uma mesma máquina poderia utilizar mais de um filme em cada período de amostragem.

- Foto: número do negativo que teve o registro fotográfico.

- Hora: horário (24h) que a foto foi registrada. Eventualmente o horário não era registrado na foto ou não era visível.

- Espécie: espécie registrada na fotografia.
- Família: família da espécie registrada na fotografia.

- Nome vulgar: nome vulgar utilizado no Rio Grande do Sul para identificar as espécies.

- Área: classificação das áreas de amostragem segundo o ambiente (Campo ou Floresta) e a distância da rodovia (Perto ou Longe).

$\mathrm{X}$ e Y: coordenadas em formato UTM, todas no Fuso 22 e datum WGS 84

Tabela 2. Número de indivíduos de espécies de mamíferos de médio e grande porte registrados na Estação Ecológica do Taim em diferentes áreas de amostragem. F | L (Floresta e Longe), F | P (Floresta e Perto), C | L (Campo e Longe), C | P (Campo e Perto).

\begin{tabular}{lccccc}
\hline Espécie & F | L & F | P & C | L & C | P & Total \\
\hline Hydrochoerus hydrochaeris & 26 & 153 & 39 & 263 & 481 \\
Lycalopex gymnocercus & 51 & 5 & 12 & & 68 \\
Cerdocyon thous & 2 & 23 & 26 & & 51 \\
Myocastor coypus & & 8 & & 21 & 29 \\
Dasypus novemcinctus & 4 & 8 & 12 & & 24 \\
Conepatus chinga & 3 & 3 & & & 6 \\
Lepus capensis & 5 & & & & 5 \\
Leopardus geoffroyi & & 2 & 2 & & 4 \\
Lontra longicaudis & & & 1 & 3 & 4 \\
Didelphis albiventris & 1 & 2 & & & 3 \\
Euphractus sexcinctus & 2 & & & & 2 \\
Mamifero não ident. & & 1 & & 1 & 2 \\
Gato doméstico & & 1 & & 1 & 2 \\
\hline Abundância & 94 & 206 & 92 & 289 & 681 \\
\hline Riqueza & 8 & 10 & 6 & 5 & 13 \\
\hline
\end{tabular}

\section{ARQUIVOS SUPLEMENTARES}

Banco de dados: Bager e Castro_dataset.

\section{AGRADECIMENTOS}

Os autores agradecem à Fundação Grupo Boticário pelo suporte financeiro para a coleta e análise dos dados. A.B. agradece à FAPEMIG pela bolsa pesquisador (2010 - 2011).

\section{REFERÊNCIAS}

NIMER, E. Climatologia do Brasil. Departamento de Recursos Naturais e Estudos Ambientais, Instituto Brasileiro de Geografia e Estatística, Rio de Janeiro, Brasil, 1989.

BAGER, A. Avaliação dos impactos das rodovias federais à fauna selvagem no extremo sul do Rio Grande do Sul-Brasil. Projeto Estrada Viva, Universidade Católica de Pelotas, Pelotas, Relatório Técnico, 2006. 
BAGER, A.; FONTOURA, V. Evaluation of the effectiveness of a wildlife roadkill mitigation system in

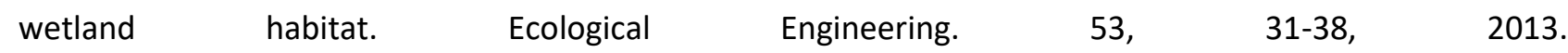
DOI.https://doi.org/10.1016/j.ecoleng.2013.01.006. 